\title{
PI3K/AKT/mTOR Signaling Pathway Regulates MMP9 Gene Activation via Transcription Factor NF-KB in Mammary Epithelial Cells of Dairy Cows
}

\author{
Yongjin Mao \\ Northeast Agricultural University \\ Chen Su \\ Northeast Agricultural University \\ Huilin Yang \\ Northeast Agricultural University \\ Feng Zhao \\ Northeast Agricultural University \\ Bo Qu \\ Northeast Agricultural University \\ Bing Zhao \\ Northeast Agricultural University \\ Yingjun Cui ( $\nabla$ c_yingjun@sina.com ) \\ Northeast Agricultural University
}

\section{Research}

Keywords: matrix metalloproteinase 9(MMP9), mammary epithelial cells, NF-kB

Posted Date: November 3rd, 2021

DOI: https://doi.org/10.21203/rs.3.rs-1029953/v1

License: () (7) This work is licensed under a Creative Commons Attribution 4.0 International License. Read Full License 


\section{Abstract \\ Background}

Matrix metalloproteinase 9 (MMP9) plays a pivotal role in mammary ductal morphogenesis, angiogenesis, and glandular tissue architecture remodeling. However, the molecular mechanism of MMP9 expression in mammary epithelial cells of dairy cows remains unclear. The current study aimed to explore the underlying mechanism of MMP9 expression.

\section{Results}

In this study, to determine whether the PI3K/AKT/mTOR/NF-KB signaling pathway participates in the regulation of MMP9 expression, we treated mammary epithelial cells with specific pharmacological inhibitors of PI3K (LY294002), mTOR (Rapamycin), or NF-KB (Celastrol), respectively. Western blotting results indicated that LY294002, Rapamycin, and Celastrol markedly decreased MMP9 expression and P65 nuclear translocation. Furthermore, we found that NF-KB (P65) overexpression resulted in elevated expression of MMP9 protein and activation of MMP9 promoter. In addition, we observed that Celastrol markedly decreases P65-overexpression-induced MMP9 promoter activity. Moreover, the results of the promoter assay indicated that the core regulation sequence for MMP9 promoter activation may be located $-80 \mathrm{bp}$ to $-420 \mathrm{bp}$ downstream from the transcription start site.

\section{Conclusions}

These observations indicated that the PI3K/AKT/mTOR signaling pathway is involved in MMP9 expression by regulating MMP9 promoter activity via NF-KB in the mammary epithelial cells of dairy cows.

\section{Introduction}

Matrix metalloproteinases (MMPs) are a multigene family of zinc-dependent endopeptidases enzymes that degrade the extracellular matrix (ECM) [1, 2]. Until now, 26 different MMPs have been confirmed in humans. Based on substrate specificity and domain homologies, MMPs were divided into six groups: collagenases, gelatinases, stromelysins, matrilysins, membrane-type MMPs, and other non-classified MMPs [3]. Typical MMP consists of several conserved domains including pre-domain, propeptide, catalytic domain, and hemopexin domain. Among these domains, the catalytic domain consists of 170 amino acids (AAs) and contains a conserved three histidine sequence which is required for zinc chelation and plays an important role in MMP activity [3].

Given their role in the degradation of the extracellular matrix, MMPs, and the plasminogen activator (PA) system may play also a critical role in the extensive remodeling that occurs in the bovine mammary gland during development, lactation, and involution [4]. The mammary gland undergoes many changes in structure and function, including cyclic expansions corresponding to the hormonal changes induced by the estrous/menstrual cycle, as well as the dramatic changes that occur during pregnancy, lactation, and involution during the lifetime of the female [5]. MMPs play a pivotal role in mammary ductal morphogenesis, angiogenesis, and glandular tissue architecture remodeling [6]. For example, remodeling of the mammary tissue is also linked with the destruction of the lobular-alveolar structure of the gland and the extracellular matrix during mammary involution [7]. At the same time, the metalloproteinase (MMP) system is activated and induces the proteolysis of extracellular matrix components [8].

Among MMPs, MMP9 has been shown to regulate many cellular processes, such as tissue repair, angiogenesis, apoptosis, cell migration, and wound healing [9]. In the mammary gland, MMP9 preferentially degrades ECM during angiogenesis and tissue remodeling. The previous study indicated that MMP9 activity is regulated at three levels: gene transcription and mRNA stability, enzymatic form activation, and inhibition by endogenous inhibitors such as tissue inhibitors of metalloproteinases (TIMPs) [10]. The MMP9 gene can be induced by a variety of oncogene products, cytokines, mitogens, and phorbol ester [11]. These stimulators can upregulate the expression of MMP9 by modulating the activation of transcription factors such as NF-KB and AP-1 through the PI3K/AKT and MAPK signaling pathways. It is well known that human MMP9 promoter contains cis-acting regulatory elements for transcription factors including an NF-KB site (located at -600bp), an SP-1 site (located at -558bp), and two AP-1 sites (located at -79bp and -533bp) [12].

Phosphatidylinositol-3-kinase is an intracellular protein with catalytic activity, which can regulate the phosphorylation of downstream Akt and constitutes the $\mathrm{PI3K} /$ Akt signaling transduction pathway [13]. The PI3K/AKT signaling pathway plays an important role in the regulation of cell survival, growth, proliferation, angiogenesis, and metabolism. In MDA-MB-231 cells, CoQ0 suppressed MMP-9 protein expression by inhibiting the PI3K/AKT/NF-KB pathway [14]. PA effectively suppressed human breast cancer MDA-MB-231 cell migration, invasion, and cell motility by abating the expression of NF-KB and AP-1 to reduce the expression of MMP-9 through ERK/PI3K/Akt/mTOR signaling pathway [15]. MMP9 secretion is mediated by 12-LOX in PC-3 cells via the activation of the $\mathrm{PISK} / \mathrm{AKT} / \mathrm{NF}-\mathrm{KB}$ signaling pathway [16].

However, the molecular mechanism governing MMP9 expression in dairy cow mammary epithelial cells has been poorly understood. In the current study, we aimed to investigate the mechanism by which PI3K/AKT/mTOR pathway is involved in NF-KB activation and MMP9 expression. We hypothesized that the $\mathrm{PI3K} / \mathrm{AKT} / \mathrm{mTOR}$ signaling pathway regulates MMP9 expression through NF-KB (P65) in mammary epithelial cells of dairy cows. To test our hypothesis, we evaluated the effects of LY294002, Rapamycin, and Celastrol on NF-KB (P65) nuclear translocation and MMP9 expression in mammary epithelial cells. We then investigated the crucial role of NF-KB(P65) in the regulation of the MMP9 promoter activity.

\section{Materials And Methods}




\section{Cell culture}

Mammary epithelial cells were isolated from mammary parenchymal tissues of lactating Holstein dairy cows as described previously [17]. All animal experimental procedures were approved by the Northeast Agricultural University Animal Care and Use Committee (Harbin, China). Mammary epithelial cells were cultured in Dulbecco's modified Eagle's medium (DMEM) supplemented with $10 \%$ fetal bovine serum (FBS), $100 \mathrm{U} / \mathrm{ml}$ penicillin, and $100 \mu \mathrm{g} / \mathrm{ml}$ streptomycin at $37^{\circ} \mathrm{C}$ in a humidified $5 \% \mathrm{CO}_{2}$ incubator. Pure mammary epithelial cells were obtained after three passages and identified by immunofluorescence for Cytokeratin-18 and $\beta$-casein [18]. All experiments were conducted using cells within seven passages.

\section{Western blotting}

Mammary epithelial cells were seeded in 6-well plates. Then cells were pre-treated with LY294002 (PI3K inhibitor), Rapamycin (mTOR inhibitor), or Celastrol (NF-KB inhibitor) for $24 \mathrm{~h}$, respectively. DMSO was used as a negative control. Whole-cell lysates were prepared from mammary epithelial cells using RIPA buffer. The total protein concentration was determined using a BCA protein assay kit (Beyotime Shanghai, Chain). Protein was separated by $10 \%$ SDS-PAGE and then transferred to nitrocellulose (NC) membranes. Immunoblot analysis was performed using antibodies against MMP9 (1:4000 dilution; Proteintech), PmTOR (1:1000 dilution; Cell Signaling Technology, Shanghai China), mTOR (1:1000 dilution; Abcam, Shanghai China), P-AKT (1:1000 dilution; Cell Signaling Technology, Shanghai China), AKT (1:1000 dilution; Cell Signaling Technology, Shanghai China), p-P65 (1:1000 dilution; Bioss, Beijing, China), P65 (1:1000 dilution; Cell Signaling Technology, Shanghai China), Histine3 (1:1000 dilution; Bioss, Beijing, China), $\beta$-actin (1:4000 dilution; Proteintech) overnight at 4 ${ }^{\circ} \mathrm{C}$. Horseradish peroxidase-conjugated IgG was used as a secondary antibody (1:10000 dilution; ZSGB-BIO, Beijing, China). The protein expression levels were detected using ECL chemiluminescence (Biosharp) and analyzed using Image J.

\section{Preparation of nuclear extract}

Mammary epithelial cells were seeded in 6-well plates. Cells were grown to 90\% confluence and treated with LY294002, Rapamycin, or Celastrol, respectively. Cells were collected after $4 \mathrm{~h}$ of treatment. Cytoplasmic and nuclear protein was prepared using Nuclear and Cytoplasmic Protein Extraction Kit (Beyotime Shanghai, Chain). The nuclear extracts and cytoplasmic extracts were stored at $-80^{\circ} \mathrm{C}$.

\section{Construction of dairy cow MMP9 promoters}

A series of fragments from the 5

- flank $\in$ gregionofthedairycowMMP9 $\geq \neq$ werepurchasedom SangonBiotech(Shanghai, Ch $\in$ a). AllMMP9 $\geq \neq$ promoteragme -end (Kpnl) and 3-end $(B g l \square)$. These MMP9 promoter agmentshadthesame3-end. Kpnl and Bgl囚 sites were included in DNA fragments so that these DNA fragments could be digested with $\mathrm{Kpnl}$ and Bgl囚, and then subcloned into the PGL3-Basic Kpnl/Bgl囚 site. The MMP9 promoter sequence was confirmed by DNA sequencing, and the resultant reporter plasmids were named MMP9-640-Luc, MMP9-420-Luc, MMP9-380-Luc, and MMP9-80-Luc respectively.

\section{Transient Transfection, and Dual-luciferase Reporter Assay}

Mammary epithelial cells were seeded in 6-well plates. Cells were grown to $80 \%$ confluence and co-transfected with $2 \mu \mathrm{g}$ of various MMP9 promoter-luciferase reporter constructs and NF-KB (P65) expression vector (purchased from Sangon Biotech) using Lipofectamine 2000 reagent (Invitrogen) according to the manufacturer's protocol. After $48 \mathrm{~h}$ of treatment, luciferase activities were determined by the Dual-Luciferase Reporter Assay System (Promega, USA) according to the manufacturer's protocol. Luciferase activity was normalized in each cell lysate to Renilla activity co-transfected as an internal control. In addition, when mammary epithelial cells were co-transfected with MMP9 promoter-luciferase reporter constructs and NF-KB(P65) expression vector for $6 \mathrm{~h}$, cells were treated with Celastrol for $48 \mathrm{~h}$, and $0.1 \%$ DMSO was used as a negative control. All transient transfections were repeated in three independent experiments.

\section{Statistical analysis}

All experiments were performed in triplicate and the results are expressed as means \pm SEM. T-test was used to compare means between two groups. One-way ANOVA followed by Tukey post hoc test was used to compare means between three groups. The minimum significance level was set at a P-value of less than 0.05 for all analyses.

\section{Results}

\section{LY294002 attenuates MMP9 expression and P65 nuclear translocation in mammary epithelial cells}

To determine whether PI3K/AKT signaling pathway is involved in MMP9 protein expression, mammary epithelial cells were treated with LY294002 (a PI3K inhibitor) for $24 \mathrm{~h}$. Compared with control, LY294002 markedly decreased the phosphorylation level of mTOR and AKT, and the expression level of MMP9 protein $(P<0.01$; Fig. 1A, B). However, there was no noticeable change in the level of AKT and mTOR protein expression under the same treatment conditions. Subsequently, we investigated whether LY294002 inhibited the translocation of NF-kB(p65) into the nucleus. Mammary epithelial cells were treated with 


\section{Rapamycin decreased MMP9 expression and P65 nuclear translocation in mammary epithelial cells}

Mammary epithelial cells were treated with Rapamycin, an mTOR inhibitor, for $24 \mathrm{~h}$. Compared with the control, treatment of mammary epithelial cells with Rapamycin tremendously decreased the expression of MMP9 and the p-mTOR protein level $(P<0.01$; Fig. 2A, B). However, the expression level of mTOR protein was not significantly changed. To investigate whether Rapamycin can affect NF-KB(p65) nuclear translocation, mammary epithelial cells were treated with Rapamycin for $4 \mathrm{~h}$. Western blot analysis showed that treatment with Rapamycin significantly increased the protein levels of P65 in the cytoplasm, and diminished nuclear P65 protein compared with the control $(P<0.01$; Fig. 2C, D).

\section{Celastrol suppressed MMP9 expression and P65 nuclear translocation in mammary epithelial cells}

To test whether MMP9 protein expression can be regulated by the transcriptional factor NF-kB in the mammary epithelial cells of dairy cows, mammary epithelial cells were treated with Celastrol (an NF-KB inhibitor) for $24 \mathrm{~h}$. We observed that Celastrol significantly decreased the expression of MMP9 compared with control $(P<0.01$; Fig. 3A, B). Next, we found that Celastrol treatment caused a marked increase in the expression of P65 in the cytosol, whereas the expression of P65 in the nucleus was significantly decreased compared with the control group $(P<0.01$; Fig. $3 \mathrm{C}, \mathrm{D})$.

\section{Transcription factor NF-kB(P65) overexpression enhanced MMP9 expression and MMP9 gene promoter activity in mammary epithelial cells}

To determine whether MMP9 expression was directly regulated by NF-kB, mammary epithelial cells were transfected with NF-kB (P65) expression vector. After transfection for 48h, we observed a significant increase in $\mathrm{P} 65$, MMP9, and p-P65 expression in the P65 overexpression group compared with the empty vector group $(P<0.05$; Fig. 4A, B). Then, the effect of transcription factor NF-KB(P65) on the transcriptional regulation of MMP9 was examined using an MMP9 promoter-luciferase construct, which contains $640 \mathrm{bp}, 420 \mathrm{bp}, 380 \mathrm{bp}$, and $80 \mathrm{bp}$ of proximal MMP9 promoter sequence upstream of a luciferase reporter. Mammary epithelial cells were transiently co-transfected with a reporter plasmid containing MMP9 promoter sequence and NF-KB(P65) expression vector for $48 \mathrm{~h}$. Our results demonstrated that the luciferase reporter gene activity of plasmid linked to the $640 \mathrm{bp}, 420 \mathrm{bp}$, and $380 \mathrm{bp}$ fragment of MMP9 promoter is significantly increased in the P65 overexpression treated group compared with the untreated group $(P<0.01 ;$ Fig. $4 \mathrm{C})$. Under the same conditions, there was no noticeable change in the M80-Luc group. Moreover, the luciferase activity of the M420-Luc group was highest in cells treated with P65 overexpression $(P<$ 0.01 ; Fig. 4C).

Next, to further determine the inhibitory effect of Celastrol on P65 overexpression-induced MMP9 gene transcription factor activity, we performed a promoter assay using transiently co-transfected mammary epithelial cells with a luciferase reporter gene linked to a $420 \mathrm{bp}$ fragment from the MMP9 promoter region and an NF-KB(P65) expression vector. After transfection for $6 \mathrm{~h}$, cells were treated with Celastrol, and DMSO was used as a negative control. Compared with the control group, treatment with Celastrol resulted in a significant decrease in the promoter activity of the MMP9 gene $(P<0.01$; Fig. 4D).

\section{Discussion}

The mammary gland is a unique glandular organ, which distinguishes mammals from all other animals and functions to produce and secrete milk to nourish offspring. The mammary gland undergoes many changes in structure and function during pregnancy, lactation, and involution. Because of the significant amount of ECM remodeling that must take place during these different stages, MMPs would be expected to play a pivotal role in mammary morphogenesis.

Previous studies have shown that PI3K/AKT signaling pathway is involved in regulating MMP9 expression [19, 20]. In the current study, we demonstrated that LY294002, a PI3K/AKT pharmacological inhibitor reported to be the first synthetic reversible PI3K inhibitor and can inhibit the catalytic activity of PI3K [21], reduced the phosphorylation of AKT and the expression of MMP9 protein. In addition, LY294002 significantly suppressed the nuclear translocation of NF-KB (P65). Similarly, LY294002 significantly decreased MMP9 protein level and AKT phosphorylation in human HCC cells [19], and NF-KB activation in MDA-MB231 human breast cancer cells[14]. Previous studies demonstrated that the PI3K/AKT pathway has a close connection with the NF-KB signaling pathway, in which the phosphorylation of Akt can activate NF-KB [22]. Inhibition of the PI3K/AKT pathway can block phosphorylation of AKT and subsequently suppress the activation of NF-KB [23]. Based our findings, we confirmed that the expression of MMP9 was regulated by PI3K/AKT signaling pathway.

The mTOR pathway is involved in many intracellular processes, such as translation and protein synthesis through its substrates [24], cell growth, and apoptosis. Moreover, PI3K and/or AKT can regulated mTOR activation [25]. In this study, we demonstrated that Rapamycin, a classic mTOR inhibitor that blocks mTOR activity through a mechanism involving an interaction with FKBP12 and a subsequent interaction with TORC1 to inhibit mTOR kinase activity [26], and suppressed expression of MMP9 in mammary epithelial cells. This agrees with the results of Park, who showed that MDA-MB-231 cells pretreated with mTOR siRNA or Rapamycin strongly inhibited MMP9 expression [15]. A more recent study demonstrated that Rapamycin decreased the expression of MMP9 and inhibited the colony formation of tumor cells in the human colorectal cancer cell line HCT116 [27]. These findings support the importance of the mTOR pathway in regulating MMP9 expression. We also showed that Rapamycin has a bigger influence on P65 nuclear translocation, and similar findings were reported by Han, who demonstrated that EPA could inhibit NF-KB (P65) translocation from the cytoplasm into the nucleus by blocking the

Page $4 / 10$ 
phosphorylation of mTOR in SKOV-3cells [28]. Previous studies also showed that AKT-dependent regulation of NF-KB is controlled by mTOR through interaction with IKK in PTEN-null/inactive prostate cancer cells [29]. It is well established that AKT controls NF-KB activation via mTOR. These results provide good evidence that NF-KB is an important downstream effector of mTOR, which is involved in MMP9 expression in mammary epithelial cells.

NF-KB is a dimeric transcription factor that regulates a target gene's expression through binding to its promoter. NF-kB is initially located in the cytoplasm in an inactive form complexed with IKB, an inhibitory factor, which binds NF-KB and masks its nuclear localization signal, thereby preventing NF-kB translocation into the nucleus $[30,31]$. Activation of NF-KB is initiated through phosphorylation of IKB by a macromolecular cytoplasmic IKB kinase (IKK). Exposure of cells to a variety of extracellular stimuli leads to the rapid phosphorylation, ubiquitination, and ultimately proteolytic degradation of $\mathrm{IKB}$, which frees $\mathrm{NF}-\mathrm{KB}$ to translocate to the nucleus where it regulates gene transcription including MMP9 [32].

Transcriptional regulation of MMP9 is believed to be the most important component for MMP9 expression. Previous studies have demonstrated that the MMP9 promoter has several transcription factor binding motifs, including NF-KB [33]. In human MCF-7 cells, NF-KB directly regulates the transcription of MMP9 [34]. Puerarin treatment effectively negated the expression of MMP9 by inhibition of the NF-KB pathway in LPS-activated MCF-7 and MDA-MB-231 cells [35]. Another study indicated that KA inhibits the PMA-induced activation of MMP-9 by suppressing NF-KB activation in HT-1080 cells [36]. In the current study, we showed that $\mathrm{P} 65$ overexpression significantly enhanced the phosphorylation level of P65 and increased MMP9 protein expression and gene promoter activity. Celastrol, an NF-KB inhibitor, remarkably reduced MMP9 protein expression and NF-KB(P65) nuclear translocation in mammary epithelial cells. Moreover, Celastrol also suppressed P65 overexpression-induced MMP9 gene promoter activity. The results of the promoter assay indicated that dairy cows' MMP9 gene promoter may contain cis-acting regulatory elements for transcription factors such as NF-KB and may be located at $-80 \sim-420 \mathrm{bp}$. It is well established that NFKB plays a significant role in the transcriptional regulation of MMP9.

Here, we examined PI3K/AKT/mTOR signaling to the MMP9 promoter in a defined system. However, the MMP9 promoter could be regulated by a network of signaling cascades because the promoter contains cis-regulatory regions that are targets of multiple signaling pathways. It remains to be seen whether $\mathrm{PI3K} / \mathrm{AKT} / \mathrm{mTOR}$ signaling can synergize or co-operate with other signaling components to further enhance MMP9 promoter activity. Therefore, further experiments will be necessary to investigate the molecular mechanism of PI3K/AKT/mTOR signaling on the transcriptional regulation of MMP9.

\section{Conclusion}

In conclusion, we show that MMP9 protein expression was mediated by transcription factor NF-KB through PI3K/AKT/mTOR signaling pathway in mammary epithelial cells of dairy cows. In addition, our study demonstrated that the core regulation sequence of transcription factor NF-KB for MMP9 promoter activation may be located in $-80 \mathrm{bp}$ to $-420 \mathrm{bp}$.

\section{Abbreviations}

MMP9

Matrix metalloproteinase 9

mTOR

mammalian target of rapamycin

DMSO

Dimethyl Sulfoxide.

\section{Declarations}

\section{Acknowledgments}

The authors would like to express special appreciation to Dr.Yingjun Cui, a professor at the College of Life Science, Key Laboratory of Dairy Science of Education Ministry, Northeast Agricultural University, for providing many insightful comments and correcting the language.

\section{Authors' contributions}

Chen Su performed experiments and carried out the statistical analysis. Yongjin and Huilin drafted the manuscript and revised the manuscript. Feng Zhao, Bo Qu, Bing Zhao, and Yingjun designed the experiments. Yingjun supervised the study and helped the revision of this manuscript. All authors read and approved the final manuscript.

\section{Funding}

This work was supported by grants from the National Natural Science Foundation of China (31401093 to Y.C., and 31571338 to F. Z.).

\section{Availability of data and materials}

The datasets used and/or analyzed during the current study are available from the corresponding authors upon reasonable request.

\section{Ethics approval and consent to participate}

All operations in this research strictly abide by the ordinances of the Administration of Laboratory Animals (College of Life Science, Northeast Agricultural University, Harbin). All animal experimental procedures were authorized by the Northeast Agricultural University Animal Care and Use Committee. 


\section{Consent for publication}

Not applicable.

\section{Competing interests}

The authors declare that they have no competing interests.

\section{References}

1. Yu HY, Kim K, Moon H, Kim K, Lee Y, Lee J. JNP3, a new compound, suppresses PMA-induced tumor cell invasion via NF-KB down regulation in MCF-7 breast cancer cells. Biochem Bioph Res Co. 2012;421(2): 190-6.

2. Overall CM, Dean RA. Degradomics: Systems biology of the protease web. Pleiotropic roles of MMPs in cancer. Cancer Metast Rev. 2006;25(1): 69-75.

3. Jabłońska-Trypuć A, Matejczyk M, Rosochacki S. Matrix metalloproteinases (MMPs), the main extracellular matrix (ECM) enzymes in collagen degradation, as a target for anticancer drugs. J Enzym Inhib Med Ch. 2016;31(sup1): 177-83.

4. Rabot A, Sinowatz F, Berisha B, Meyer HHD, Schams D. Expression and localization of extracellular Matrix-Degrading proteinases and their inhibitors in the bovine mammary gland during development, function, and involution. J Dairy Sci. 2007;90(2): 740-8.

5. Inman JL, Robertson C, Mott JD, Bissell MJ. Mammary gland development: Cell fate specification, stem cells and the microenvironment. Development. 2015;142(6): 1028-42.

6. Vu TH, Werb Z. Matrix metalloproteinases: Effectors of development and normal physiology. Genes Dev. 2000;14(17): 2123-33.

7. Boutinaud M, Isaka N, Gandemer E, Lamberton P, Wiart S, Taranilla AIDP et al. Inhibiting prolactin by cabergoline accelerates mammary gland remodeling during the early dry period in dairy cows. J Dairy Sci. 2017;100(12): 9787-98.

8. Tremblay G, Bernier-Dodier P, Delbecchi L, Wagner GF, Talbot BG, Lacasse P. Local control of mammary involution: Is stanniocalcin-1 involved? J Dairy Sci. 2009;92(5): 1998-2006.

9. Nemeth JA. Matrix metalloproteinase activity, bone matrix turnover, and tumor cell proliferation in prostate cancer bone metastasis. CancerSpectrum Knowledge Environment. 2002;94(1): 17-25.

10. Yan C, Boyd DD. Regulation of matrix metalloproteinase gene expression. J Cell Physiol. 2007;211(1): 19-26.

11. Curran S, Murray GI. Matrix metalloproteinases in tumour invasion and metastasis. The Journal of Pathology. 1999;189(3): 300-8.

12. Cho H, Jeong Y, Park K, Park Y, Chung I, Lee K et al. Bee venom suppresses PMA-mediated MMP-9 gene activation via JNK/p38 and NF-KB-dependent mechanisms. J Ethnopharmacol. 2010;127(3): 662-8.

13. Sun L, Tian W, Guo X, Zhang Y, Liu X, Li X et al. Lactobacillus gasseri JM1 with potential probiotic characteristics alleviates inflammatory response by activating the PI3K/Akt signaling pathway in vitro. J Dairy Sci. 2020;103(9): 7851-64.

14. Yang $\mathrm{H}$, Thiyagarajan V, Shen P, Mathew DC, Lin K, Liao J et al. Anti-EMT properties of CoQ0 attributed to PI3K/AKT/NF-KB/MMP-9 signaling pathway through ROS-mediated apoptosis. J Exp Clin Canc Res. 2019;38(1).

15. Park JH, Cho YY, Yoon SW, Park B. Suppression of MMP-9 and FAK expression by pomolic acid via blocking of NF-kappaB/ERK/mTOR signaling pathways in growth factor-stimulated human breast cancer cells. Int J Oncol. 2016;49(3): 1230-40.

16. Dilly A, Ekambaram P, Guo Y, Cai Y, Tucker SC, Fridman R et al. Platelet-type 12-lipoxygenase induces MMP9 expression and cellular invasion via activation of PI3K/Akt/NF-KB. Int J Cancer. 2013;133(8): 1784-91.

17. Cui Y, Liu Z, Sun X, Hou X, Qu B, Zhao F et al. Thyroid hormone responsive protein spot 14 enhances lipogenesis in bovine mammary epithelial cells. In Vitro Cellular \& Developmental Biology - Animal. 2015;51(6): 586-94.

18. Cui Y, Sun X, Jin L, Yu G, Li Q, Gao X et al. MiR-139 suppresses $\beta$-casein synthesis and proliferation in bovine mammary epithelial cells by targeting the GHR and IGF1R signaling pathways. Bmc Vet Res. 2017;13(1).

19. Tian T, Nan KJ, Guo H, Wang WJ, Ruan ZP, Wang SH et al. PTEN inhibits the migration and invasion of HepG2 cells by coordinately decreasing MMP expression via the PI3K/Akt pathway. Oncol Rep. 2010;23(6): 1593-600.

20. Jung J, Jung K, Kim D, Kim H. Selective inhibition of MMP-9 gene expression by mangiferin in PMA-stimulated human astroglioma cells: Involvement of PI3K/Akt and MAPK signaling pathways. Pharmacol Res. 2012;66(1): 95-103.

21. Sheridan C, Downward J. Inhibiting the RAS-PI3K pathway in cancer therapy. Enzymes. 2013;34 Pt. B(107-36.

22. Lee $\mathrm{Y}$, Lin H, Hsu C, Wang C, Chiang T, Chen J. Inhibitory effects of andrographolide on migration and invasion in human non-small cell lung cancer A549 cells via down-regulation of PI3K/Akt signaling pathway. Eur J Pharmacol. 2010;632(1-3): 23-32.

23. Jiang R, Xu J, Zhu D, Li J, Zhang C, Lin N et al. Glycyrrhizin inhibits osteoarthritis development through suppressing the PI3K/AKT/NF-KB signaling pathwayin vivo and in vitro. Food Funct. 2020;11(3): 2126-36.

24. Sipka AS, Chandler TL, Behling-Kelly EL, Overton TR, Mann S. The effect of ex vivo lipopolysaccharide stimulation and nutrient availability on transition cow innate immune cell AKT/mTOR pathway responsiveness. J Dairy Sci. 2020;103(2): 1956-68.

25. Laplante M, Sabatini DM. MTOR signaling in growth control and disease. Cell. 2012;149(2): 274-93.

26. Sawyers CL. Will mTOR inhibitors make it as cancer drugs? Cancer Cell. 2003;4(5): 343-8.

27. Zhang ZH, Li MY, Wang Z, Zuo HX, Wang JY, Xing Y et al. Convallatoxin promotes apoptosis and inhibits proliferation and angiogenesis through crosstalk between JAK2/STAT3 (T705) and mTOR/STAT3 (S727) signaling pathways in colorectal cancer. Phytomedicine. 2020;68(153172. 
28. Han L, Zhang Y, Meng M, Cheng D, Wang C. Eicosapentaenoic acid induced SKOV-3 cell apoptosis through ERK1/2-mTOR-NF-KB pathways. Anti-Cancer Drug. 2016;27(7): 635-42.

29. Dan HC, Cooper MJ, Cogswell PC, Duncan JA, Ting JPY, Baldwin AS. Akt-dependent regulation of NF-kB is controlled by mTOR and Raptor in association with IKK. Gene Dev. 2008;22(11): 1490-500.

30. Noh E, Youn HJ, Jung SH, Han J, Jeong Y, Chung E et al. Cordycepin inhibits TPA-induced matrix metalloproteinase-9 expression by suppressing the MAPK/AP-1 pathway in MCF-7 human breast cancer cells. Int J Mol Med. 2010;25(2): 255.

31. JIANG Q, PAN YU, CHENG Y, LI H, LIU D, LI H. Lunasin suppresses the migration and invasion of breast cancer cells by inhibiting matrix metalloproteinase2/-9 via the FAK/Akt/ERK and NF-KB signaling pathways. Oncol Rep. 2016;36(1): 253-62.

32. Karin M, Ben-Neriah Y. Phosphorylation meets ubiquitination: The control of NF-KB activity. Annu Rev Immunol. 2000;18(1): 621-63.

33. Himelstein BP, Lee EJ, Sato H, Seiki M, Muschel RJ. Tumor cell contact mediated transcriptional activation of the fibroblast matrix metalloproteinase-9 gene: Involvement of multiple transcription factors including Ets and an alternating purine-pyrimidine repeat. Clin Exp Metastas. 1998;16(2): $169-77$.

34. Ricca A, Biroccio A, Del BD, Mackay AR, Santoni A, Cippitelli M. Bcl-2 over-expression enhances NF-kappaB activity and induces mmp-9 transcription in human MCF7(ADR) breast-cancer cells. Int J Cancer. 2000;86(2): 188-96.

35. Liu X, Zhao W, Wang W, Lin S, Yang L. Puerarin suppresses LPS-induced breast cancer cell migration, invasion and adhesion by blockage NF-kB and Erk pathway. Biomed Pharmacother. 2017;92(429-36.

36. Choi JH, Hwang YP, Jin SW, Lee GH, Kim HG, Han EH et al. Suppression of PMA-induced human fibrosarcoma HT-1080 invasion and metastasis by kahweol via inhibiting Akt/JNK1/2/p38 MAPK signal pathway and NF-KB dependent transcriptional activities. Food Chem Toxicol. 2019;125(1-9.

\section{Figures}

A

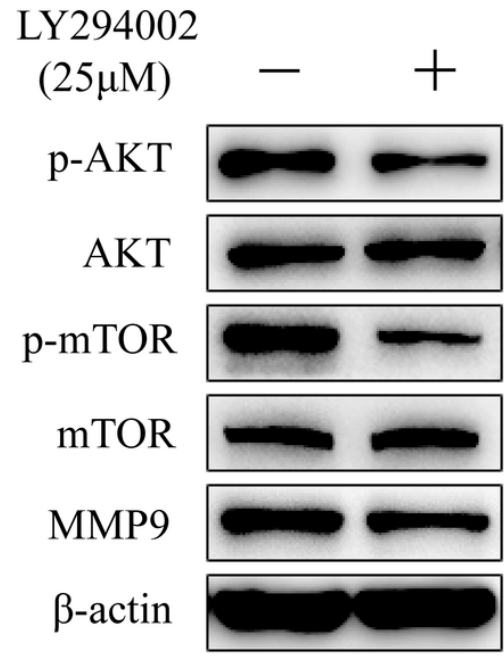

$\mathrm{C}$

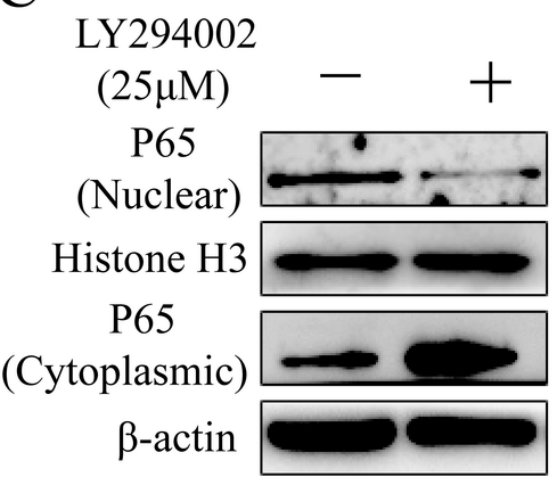

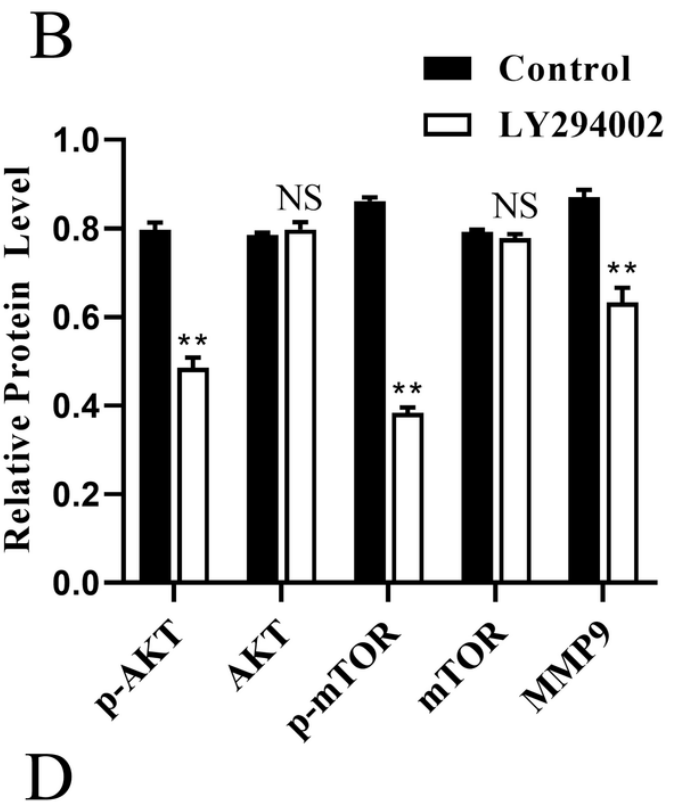

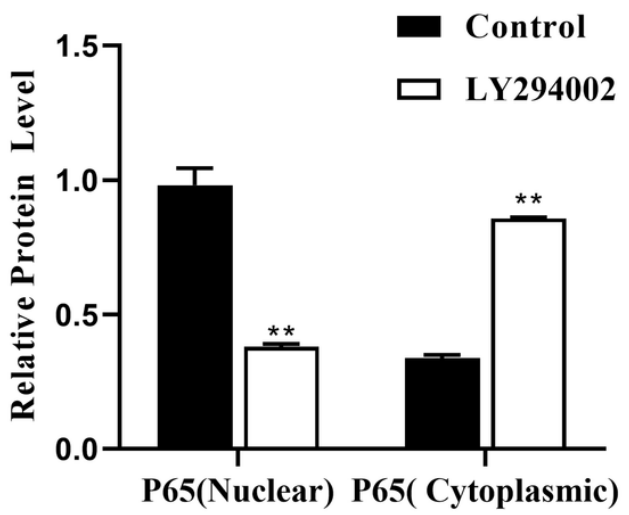

Figure 1

LY294002 attenuates MMP9 expression and P65 nuclear translocation in mammary epithelial cells. (A) Mammary epithelial cells were pretreated with $25 \mu \mathrm{M}$ LY294002 (a selective antagonist of PI3K/AKT) for 24h. DMSO was used as a negative control. The expression of P-AKT, AKT, P-mTOR, mTOR MMP9 was measured by Western blot analysis. $\beta$-actin expression was evaluated as an internal control. (B) Quantification of P-AKT, AKT, P-mTOR, mTOR, and MMP9 expression from the Western blots in panel A. (C) Mammary epithelial cells were treated with $25 \mu \mathrm{M}$ LY294002 for $4 \mathrm{~h}$. Nuclear and cytoplasmic extracts were 
prepared, and Western blot analysis was performed. Histone $\mathrm{H} 3$ and $\beta$-actin were used as an internal control for nuclear and cytoplasmic extracts respectively. (D) Quantification of nuclear and cytoplasmic P65 expression from the Western blots in panel C. One representative image of three different experiments for each of the analyses performed is shown. Data are expressed as mean \pm SEM of three independent experiments. ${ }^{*} \mathrm{P}<0.05$ and ${ }^{* * P}<0.01$ compared with the control.

A

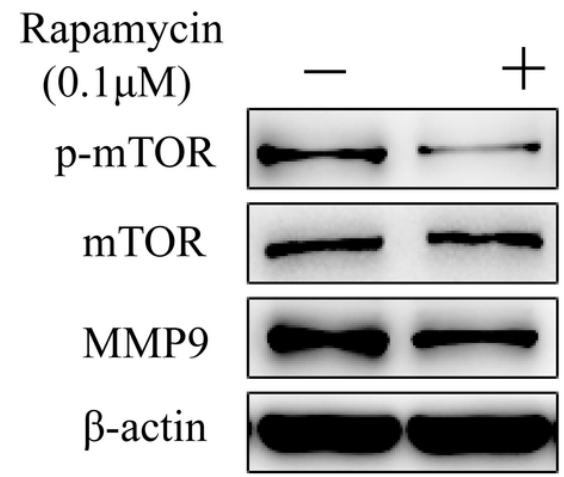

C

\section{Rapamycin}

$(0.1 \mu \mathrm{M})$ P65

(Nuclear)

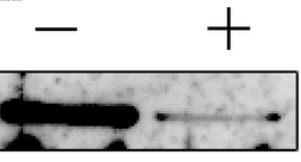

\section{Histone H3}

\section{P65}

(Cytoplasmic)

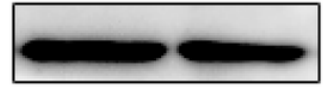

$\beta$-actin
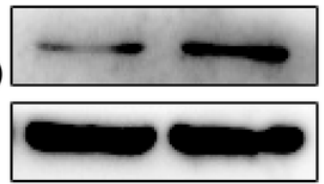
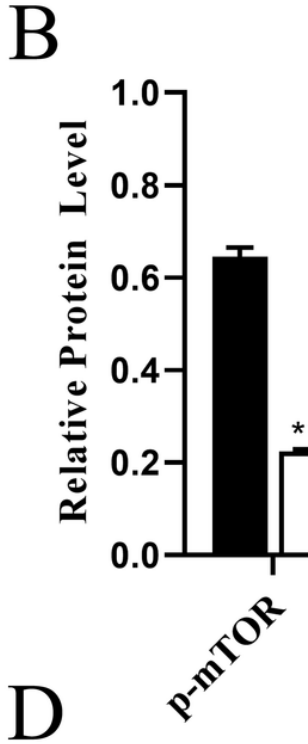

\section{$\square$ Rapamycin}

Control

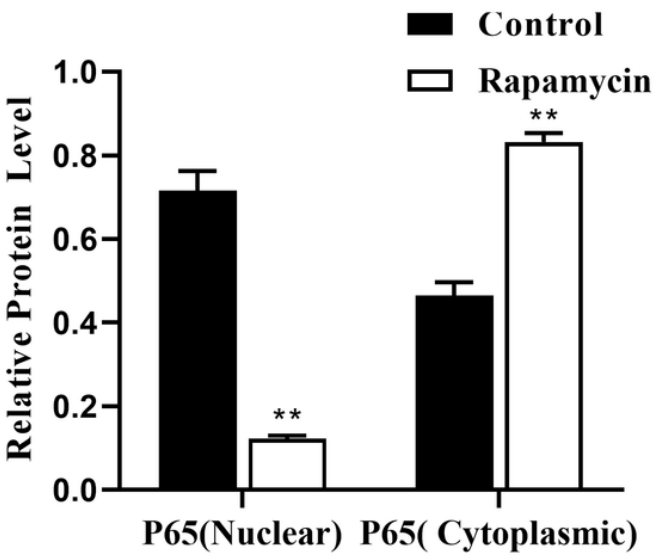

Figure 2

Rapamycin decreased MMP9 expression and P65 nuclear translocation in mammary epithelial cells. (A) Mammary epithelial cells were pretreated with $0.1 \mu \mathrm{M}$ Rapamycin (an mTOR inhibitor) for 24h. The expression of P-mTOR, mTOR, and MMP9 was measured by Western blot analysis. $\beta$-actin expression was evaluated as an internal control. (B) Quantification of P-mTOR, mTOR, and MMP9 expression from the Western blots in panel A. (C) Mammary epithelial cells were treated with $0.1 \mu \mathrm{M}$ Rapamycin for $4 \mathrm{~h}$. Nuclear and cytoplasmic extracts were prepared, and Western blot analysis was performed. Histone $\mathrm{H} 3$ and $\beta$-actin were used as an internal control for nuclear and cytoplasmic extracts, respectively. (D) Quantification of nuclear and cytoplasmic P65 expression from the Western blots in panel C. One representative image of three different experiments for each of the analyses performed is shown. Data are expressed as mean \pm SEM of three independent experiments. ${ }^{*} \mathrm{P}<0.05$ and ${ }^{*} \mathrm{P}<0.01$ compared with the control. 


\section{B}

Celastrol

$(0.3 \mu \mathrm{M})$

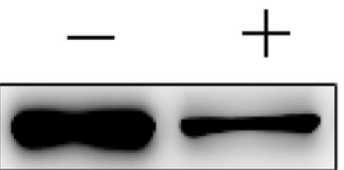

MMP9

$\beta$-actin $\square$

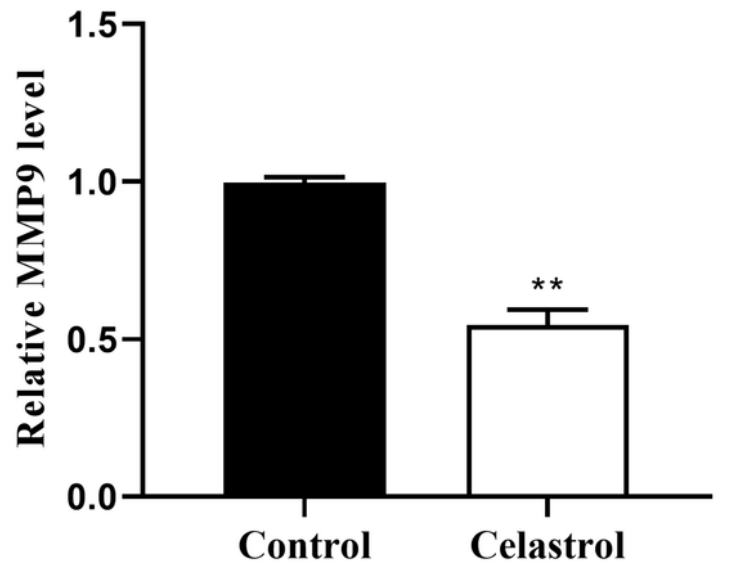

C

Celastrol

$(25 \mu \mathrm{M})$

P65

(Nuclear)

Histone H3

P65

(Cytoplasmic)

$\beta$-actin
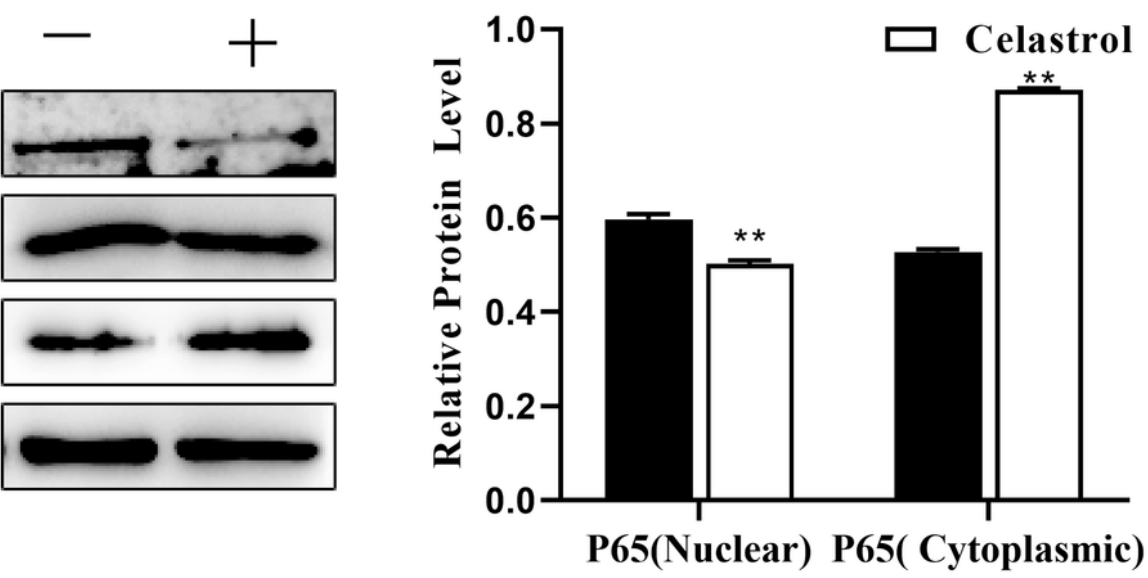

Figure 3

Celastrol suppressed MMP9 expression and P65 nuclear translocation in mammary epithelial cells. (A) Mammary epithelial cells were pretreated with $0.3 \mu \mathrm{M}$ Celastrol (an NF-KB inhibitor) for 24h. The expression of MMP9 was measured by Western blot analysis. $\beta$-actin expression was evaluated as an internal control. (B) Quantification of MMP9 expression from the Western blots in panel A. (C) Mammary epithelial cells were treated with $0.3 \mu \mathrm{M}$ Celastrol for $4 \mathrm{~h}$. Nuclear and cytoplasmic extracts were prepared and Western blot analysis was performed. Histone $\mathrm{H} 3$ and $\beta$-actin were used as an internal control for nuclear and cytoplasmic extracts respectively. (D) Quantification of nuclear and cytoplasmic P65 expression from the Western blots in panel C. One representative image of three different experiments, for each of the analyses performed, is shown. Data are expressed as mean $\pm S E M$ of three independent experiments. ${ }^{*}<<$ 0.05 and ${ }^{* *} \mathrm{P}<0.01$ compared with the control. 
A
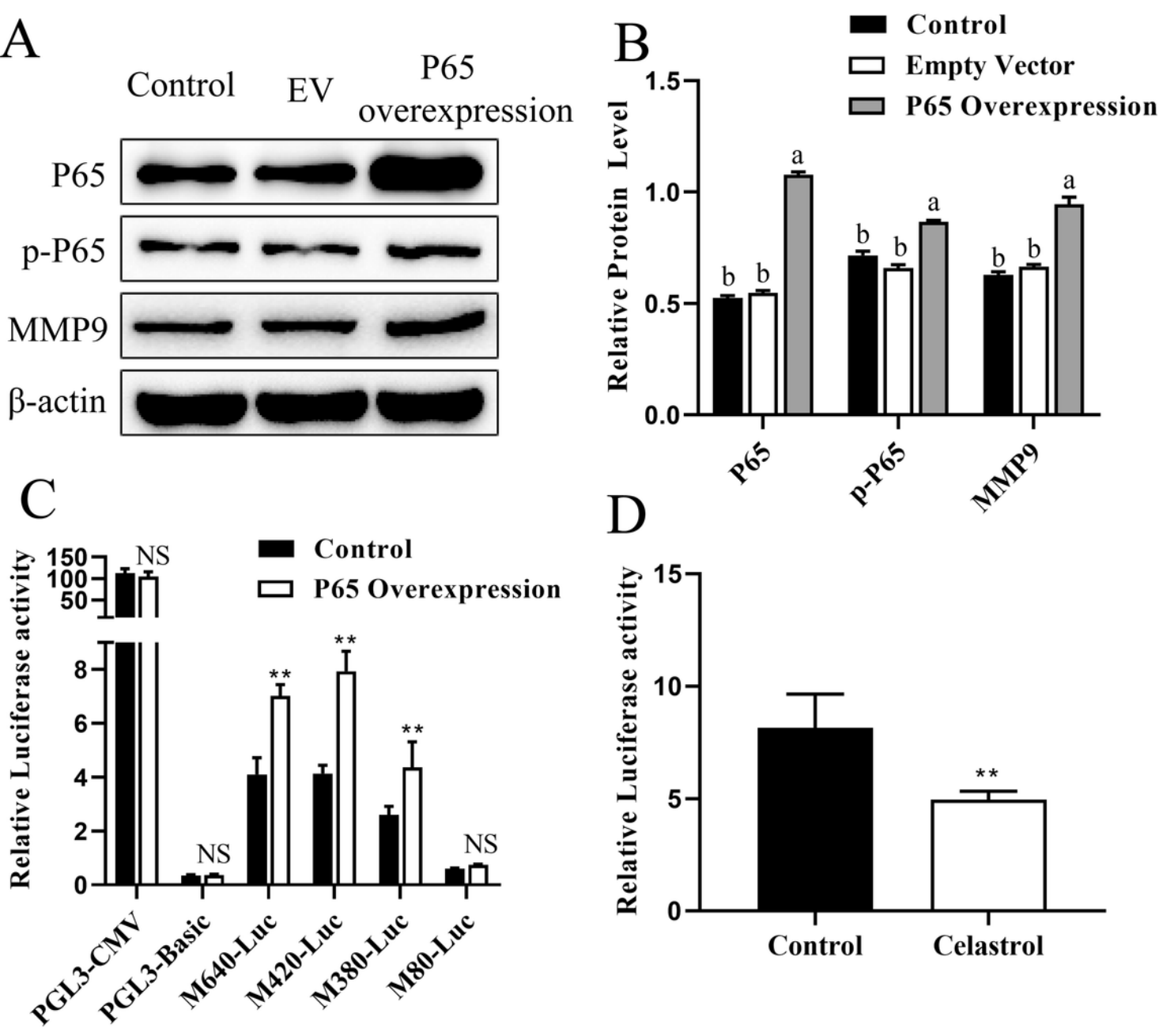

Figure 4

Effect of transcription factor NF-KB (P65) on MMP9 promoter activity in mammary epithelial cells. (A) Mammary epithelial cells were transfected with NF-KB (P65) expression vector for 48h. The levels of P65, P-P65, and MMP9 were determined by Western blotting. Protein expression levels of $\beta$-actin in cell lysates were used as a control. (B) Quantification of P65, P-P65, and MMP9 expression from the Western blots in panel A. (C) Mammary epithelial cells were cotransfected with a series of MMP9-PGL3 plasmid including MMP9-640-Luc (M640-Luc), MMP9-420-Luc (M420-Luc), MMP9-380-Luc (M380-Luc), MMP9-80Luc (M80-Luc), which contains a deletion of the MMP9 gene promoter 5 -flanking region, and NF-KB (P65) overexpression vector for 48h. Luciferase activity was normalized to the Renilla activity in each cell lysate. (D) Mammary epithelial cells were co-transfected with M420-Luc plasmid and NF-KB(P65) overexpression vector for $6 \mathrm{~h}$. Then cells were treated with Celastrol, and DMSO was used as a negative control. Luciferase activity was normalized with the Renilla activity in each cell. Data are expressed as mean \pm SEM of three independent experiments. For $B$, means without a common letter (a-b) differ at $P<$ 0.05. For $C$ and $D, * P<0.05 ; * * P<0.01$. 